\title{
SEROPREVALENCIA DE LEPTOSPIROSIS EN PUENTE PIEDRA, LIMA EN EL AÑO 2006
}

\author{
James A. Platts-Mills ${ }^{1, a}$, Patrick LaRochelle, ${ }^{1, a}$, Kalina Campos ${ }^{2, b}$, Joseph M. Vinetz ${ }^{2,3, a}$, \\ Eduardo Gotuzzo,a , Jessica N. Ricaldi ${ }^{2, a, c}$
}

\begin{abstract}
RESUMEN
La leptospirosis es una enfermedad ampliamente prevalente en áreas tropicales, pero también se presenta en áreas urbanas. El presente estudio tiene como objetivo determinar la seroprevalencia para Leptospira en el distrito de Puente Piedra, donde se han presentado casos de leptospirosis severa en los últimos años. Se recolectaron datos relacionados a factores de riesgo asociados con leptospirosis y muestras de sangre de 250 participantes, seleccionados por un muestreo aleatorio. Se encontró una alta prevalencia de factores de riesgo en la población y, usando la prueba de aglutinación microscópica, anticuerpos circulantes contra Leptospira en solamente tres participantes $(1,2 \%)$.
\end{abstract}

Palabras clave: Leptospirosis; Seroprevalencia; Estudios epidemiológicos; Perú (fuente: DeCS BIREME).

\section{SEROPREVALENCE OF LEPTOSPIROSIS IN PUENTE PIEDRA, LIMA, IN 2006}

\begin{abstract}
Leptospirosis is a disease widely prevalent in tropical areas, but may also be present in urban areas. The present study aims to determine the seroprevalence of Leptospira in the district of Puente Piedra, where there have been cases of severe leptospirosis in recent years. We collected data related to risk factors associated with leptospirosis and blood samples from 250 participants, selected by random sampling. We found a high prevalence of risk factors in the population and using the microscopic agglutination test, antibodies were found in only 3 participants $(1.2 \%)$.
\end{abstract}

Key words: Leptospirosis; Seroprevalence; Seroepidemiologic studies; Peru (source: MeSH NLM).

\section{INTRODUCCIÓN}

La leptospirosis es una enfermedad zoonótica de distribución mundial que se presenta tanto de forma endémica como epidémica ${ }^{(1)}$. En los últimos años se han descrito con mayor frecuencia casos aislados y epidemias en áreas urbanas y periurbanas. Muchas de estas áreas tienen condiciones deficientes de salubridad que favorecen la transmisión de leptospirosis, entre ellas tenemos a las llamadas "favelas" o "pueblos jóvenes" que surgen alrededor de las ciudades grandes en América Latina ${ }^{(2,3)}$. La transmisión de leptospirosis ocurre por medio del contacto con aguas y suelos contaminados por la orina de ratas, perros y otros animales portadores ${ }^{(1)}$. EI riesgo de transmisión aumenta cuando ocurren fenómenos naturales como lluvias e inundaciones.

La presentación clínica de la infección por especies patógenas de Leptospira es muy variable. Las infecciones asintomáticas son comunes y en la mayoría de casos los síntomas se limitan a una fiebre indiferenciada. Sin embargo, hasta el $10 \%$ de casos sintomáticos presenta manifestaciones graves como la enfermedad de Weil, hemorragia pulmonar, miocarditis o meningitis aséptica (1). Los factores de riesgo para el desarrollo de una infección grave aún no se han dilucidado ${ }^{(4)}$.

En el Perú se han descrito brotes epidémicos de leptospirosis en la costa, sierra y selva. El número de casos se ha incrementado en los últimos años, especialmente en la selva alta y selva baja del país, aunque este incremento puede reflejar solo un mayor acceso a pruebas diagnósticas. En Lima se han reportado casos dentro del área metropolitana, especialmente en las áreas cercanas al río Rímac ${ }^{(5)}$. En el Hospital Cayetano Heredia en los últimos años se han diagnosticado múltiples casos graves provenientes del área cercana al río Chillón, incluyendo el área de Puente Piedra (datos

\footnotetext{
University of Virginia. Charlottesville, Estados Unidos de Norteamérica.

2 Universidad Peruana Cayetano Heredia. Lima, Perú

3 University of California. San Diego, Estados Unidos de Norteamérica.

a Médico; ${ }^{b}$ Bióloga; ${ }^{c}$ Doctor (PhD) en Patología Molecular.
}

Recibido: 15-10-10 Aprobado: 18-05-11 
no publicados). La existencia de estos casos sugiere un posible foco de transmisión de leptospirosis en el área.

Puente Piedra es un distrito al norte de Lima con una superficie de $71,18 \mathrm{~km}^{2}$ que limita por el sur con el río Chillón. El acceso a servicios básicos no es uniforme, en el 2006 el 38,66 \% de viviendas de este distrito tenían paredes de material rústico, el $91,33 \%$ de hogares contaba con energía eléctrica, pero solo el $37,53 \%$ contaba con acceso a agua de la red pública y el 30,46\% contaba con sistema de desagüe. El $65,10 \%$ de la población es económicamente activa y tiene un promedio de 9,2 años de escolaridad (6).

Se llevó a cabo una encuesta seroepidemiológica de julio a septiembre de 2006 para evaluar la prevalencia de leptospirosis, identificar los serovares reactivos y conocer factores asociados con seropositividad en Puente Piedra.

\section{EL ESTUDIO}

Para este estudio se calculó una muestra de 250 individuos, con un nivel de confianza de $95 \%$ y un intervalo de error de $2,7 \%$. Para este cálculo se consideró que la población total de Puente Piedra era 203473 habitantes ${ }^{(7)}$; se estimó una prevalencia del $5 \%$ basándose en estudios que muestran un rango de seroprevalencia de $0,7 \%$ (Pampas de San Juan) a 10\% (Chancay) en el departamento de Lima ${ }^{(3)}$.

Se utilizó un generador de números aleatorios para elegir 250 manzanas residenciales, delineadas en el mapa usado para el Censo Nacional 2005 por el INEI (Instituto Nacional de Estadística e Informática). En cada manzana, se eligió un lado y una puerta lanzando una moneda al aire y tirando un dado, respectivamente. Para fines de este estudio la población se estratificó por edad y sexo en seis grupos (hombres y mujeres de 7-19, 20-35 y >35 años). Se buscó un grupo por cada manzana y se alternó sucesivamente. Si no se encontró un participante que cumpliera criterios de inclusión o si se negó a participar, la búsqueda se continuó en el sentido de las agujas del reloj.

Se recolectaron datos sobre factores de riesgo que sugieran exposición a Leptospira y se tomó una muestra de sangre de $5 \mathrm{~mL}$ por participante. La muestra de sangre se centrifugó el mismo día y el suero fue conservado a $-20{ }^{\circ} \mathrm{C}$ hasta su análisis. Todos los voluntarios firmaron un consentimiento informado antes de participar en este estudio, aprobado por los comités de ética de la Universidad de Virginia en los Estados Unidos y de la Universidad Peruana Cayetano Heredia en Lima. Los datos y las muestras de los voluntarios fueron codificados para mantener la confidencialidad del estudio.
Se buscó evidencia de infección con Leptospira usando la prueba de microaglutinación (MAT), el estándar de oro para el diagnóstico. Para la realización de esta prueba se usan como antígeno leptospiras cultivadas en medio líquido. Para este estudio se utilizaron los siguientes serovares: Alexi, Australis, Autumnalis, Ballum, Bataviae, Boricana, Bratislava, Canicola, Celledoni, Copenhageni, Cynopteri, Djasiman, Georgia, Grippotyphosa, Icterohaemorrhagiae, Javanica, Mankarso, Panamá, Pomona, Tarassovi, Varillal y Wolfii,

Si se observa una aglutinación igual o mayor al 50\% de las leptospiras con al menos uno de los serovares en una dilución del suero de 1:50 o más la prueba se consideró positiva. En los sueros positivos se realizaron diluciones seriadas.

Se utilizó el programa SPSS 14 para Windows para crear una base de datos con la información epidemiológica y clínica de los voluntarios y para el análisis estadístico.

\section{HALLAZGOS}

En la población de estudio se encontró una amplia prevalencia de factores que podrían causar exposición a Leptospira, incluyendo: tener uno o más perros en casa $(62,8 \%)$, no tener agua y desagüe de la red pública (31,2 y $62,4 \%$ respectivamente), tener animales que orinan dentro de la casa $(42,4 \%)$, la presencia de roedores $(29,2 \%$ los ve $>1$ vez/semana en el hogar), contacto con agua de río (14\%) y tener contacto con agua, lodo o tierra en el trabajo $(14,02 \%)$.

Tres de las 250 muestras de suero obtenidas fueron positivas $(1,2 \% ; 95 \% \mathrm{Cl} 0,24-3,6 \%)$, con un título de MAT de 1:50 o más para uno de los serovares. Una de las muestras tuvo títulos de 1:50 contra el serovar $\mathrm{Ce}$ lledoni y 1:200 contra el serovar Varillal, las otras dos muestras tuvieron títulos de 1:50 contra el serovar CeIledoni. Los tres sujetos positivos fueron varones (test exacto de Fisher $p=0,07$ ) y tenían 28, 50, y 67 años. Ninguno de los tres tenía una historia de fiebre con o sin ictericia en el año anterior, y ninguno había viajado a lugares donde la leptospirosis es endémica. Todos ellos negaron formas de exposición laboral, trabajo del campo o irrigación e, incluso, contacto con animales o posibles fuentes de agua contaminada. Dos de los tres sujetos con serología positiva usaban una cisterna como la fuente principal de agua, los tres tenían perros que vivían en su casa $(p=0,29)$ y dos de los tres reportaban la presencia de roedores en su casa durante el mes previo $(p=0,57)$. 


\section{DISCUSIÓN}

Nuestros resultados, sorprendentemente, muestran una seroprevalencia baja para leptospiras en Puente Piedra en el año 2006. Considerando el antecedente de casos graves y que en la población de estudio existe una prevalencia alta de múltiples factores de riesgo para la exposición a Leptospira, incluyendo condiciones de salubridad deficientes, el contacto con orina de animales y la presencia de roedores, se esperaba una seroprevalencia más alta.

Se ha descrito que al existir reacciones cruzadas entre serogrupos, el serovar aglutinante en MAT no predice el serogrupo infectante ${ }^{(8)}$, por lo cual no podemos hacer inferencias con respecto a posibles fuentes de transmisión, a pesar de que el serovar Varillal se ha aislado en ratas en Iquitos y el serovar Celledoni se ha aislado de ratas y ganado vacuno en diversas partes del mundo ${ }^{(9)}$.

El presente estudio tiene varias limitaciones. Nuestra estrategia de muestreo dificultó el enrolamiento de varones, que a menudo trabajan fuera de la casa durante el día, por lo cual es posible que se haya subestimado la prevalencia de la exposición. Además, los brotes de leptospirosis se pueden asociar con una fuente de exposición acuática y es posible que nuestra muestra no lograra identificar el área con mayor prevalencia dentro del distrito. También es posible que personas que desarrollaron títulos bajos de anticuerpos durante la época de inundaciones hayan seroconvertido y sean negativos al momento del muestreo. Una posibilidad mas alejada es que el serovar infectante en el área aún no haya sido aislado e identificado $y$, por lo tanto, no forma parte del panel de prueba, por lo cual no se podría detectar. Un fenómeno similar ocurrió en el área alrededor de lquitos, donde se aisló una especie nueva de Leptospira y se encontró una alta seroprevalencia únicamente a esta especie en la población de la zona ${ }^{(9)}$. Finalmente, solo uno de los voluntarios con serología positiva vivía en una zona cercana al río Chillón, sin embargo, al no haberse incluido dentro del estudio una pregunta específica al respecto, no podemos analizar esta variable.

La ciudad de Lima tiene un clima desértico y es atravesada por dos ríos. El caudal de estos ríos se incrementa en los meses de diciembre a marzo debido a un aumento en las precipitaciones fluviales en áreas afluentes. Con frecuencia ocurren desbordes e inundaciones en el área circundante al río Chillón en esta época del año. Muchos brotes periurbanos de leptospirosis ocurren en el contexto de lluvias fuertes, las que causan desbordes de ríos, canales y alcantarillas, contaminación del agua potable y mayor exposición a focos de la infección, incluso a ratas ${ }^{(10,11)}$. Estudios realizados en la Amazonía peruana han demostrado que se pueden cuantificar la carga de leptospiras en fuentes de agua (ríos, canales, pozos) y que esta es altamente variable inclusive en días consecutivos ${ }^{(12)}$, lo cual puede dificultar la identificación del foco transmisor.

El potencial de brotes epidémicos en áreas cercanas a ríos y canales hace que sea muy importante tener un alto índice de sospecha en el contexto clínico apropiado, especialmente durante de la temporada de lluvias. Para evaluar más a fondo la prevalencia real de leptospirosis en las zonas de riesgo, será necesario determinar la prevalencia de infección en animales y evaluar el riesgo de infección a partir de fuentes de agua utilizando métodos moleculares ${ }^{(12)}$.

\section{AGRADECIMIENTOS}

A las Dras. Elsa Gonzales y Mayuko Saito por sus sugerencias para el diseño del presente estudio. Al Dr. Humberto Guerra por la revisión crítica del manuscrito.

\section{Contribuciones de Autoría}

JP y PL diseñaron el estudio, recolectaron muestras, analizaron datos y redactaron el manuscrito. $\mathrm{KC}$ realizó el análisis de las muestras en el laboratorio. JV y EG contribuyeron en el diseño el estudio, hicieron una revisión crítica del manuscrito y aprobaron el manuscrito final. JR redactó el manuscrito, analizó e interpretó datos y aprobó el manuscrito final.

\section{Conflictos de Interés}

Los autores no reportan conflictos de interés.

\section{REFERENCIAS BIBLIOGRÁFICAS}

1. Bharti AR, Nally JE, Ricaldi JN, Matthias MA, Díaz MM, Lovet MA et al; Peru-United States Leptospirosis Consortium. Leptospirosis: a zoonotic disease of global importance. Lancet Infect Dis. 2003;3:757-71.

2. Vinetz JM, Glass GE, Flexner CE, Mueller P, KasIow DC. Sporadic urban leptospirosis. Ann Intern Med. 1996;125:794-8.

3. Johnson MA, Smith H, Joeph P, Gilman RH, Bautista CT, Campos KJ et al. Environmental exposure and leptospirosis, Peru. Emerg Infect Dis. 2004;10:1016-22.

4. Cachay ER, Vinetz JM. A global research agenda for leptospirosis. J Postgrad Med. 2005;51:174-8.

5. Céspedes M, Balda L, González D, Tapia R. Situación de la leptospirosis en el Perú 1994-2004. Rev Peru Med Exp Salud Publica. 2006;23(1):56-66.

6. Ministerio de Salud del Perú, Oficina de Epidemiología. Sala situacional S.B.S. Puente piedra S. E. $32-2008$. 
Lima: MINSA; 2008. Disponible en: http://www.disavlc. gob.pe/portal/index.php?option=com_docman\&task=doc_ download\&gid=229\&ltemid=34

7. Instituto Nacional de Estadistica e Informatica (INEI): Censos Nacionales 2005. Lima: INEI; 2006. Disponible en : http://www1.inei.gob.pe/web/NotaPrensaFlotante. asp?file=6190.pdf. Fecha de acceso: Abril 27, 2011.

8. Levett PN. Usefulness of serologic analysis as a predictor of the infecting serovar in patients with severe leptospirosis. Clin Infect Dis. 2003;36(4):447-52.

9. Matthias MA, Ricaldi JN, Cespedes M, Diaz MM, Galloway RL, Saito $\mathbf{M}$ et al. Human leptospirosis caused by a new, antigenically unique Leptospira associated with a Rattus species reservoir in the Peruvian Amazon. PLOS Neglected Diseases. 2008;2:e213.

10. Dias JP, Teixeira MG, Costa MC, Mendes CM, Guimaraes $\mathbf{P}$, Reis MG et al. Factors associated with Leptospira $\mathrm{sp}$ infection in a large urban center in northeastern Brazil. Rev Soc Bras Med Trop. 2007;40:499-504.
11. Sarkar U, Nascimento SF, Barbosa R, et al. Populationbased case-control investigation of risk factors for leptospirosis during an urban epidemic. Am J Trop Med Hyg. 2002;66:605-10.

12. Ganoza CA, Matthias MA, Collins-Richards D, Brouwer $\mathrm{KC}$, Cunningham CB, Segura ER et al. Determining risk for severe leptospirosis by molecular analysis of environmental surface waters for pathogenic Leptospira. PLoS Med. 2006;3(8):e308.

Correspondencia: Joseph M Vinetz

Dirección: Universidad de California San Diego

9500 Gilman Drive \# 0741, La Jolla, CA 92093

Teléfono: 1 (858) 822-4469

Correo electrónico: jvinetz@ucsd.edu

\section{Suscríbase en forma electrónica y gratuita a los contenidos de la Revista Peruana de Medicina Experimental y Salud Pública, ingrese a WWW.ins.gob.pe, seleccione el ícono de la revista y envíenos sus datos.}

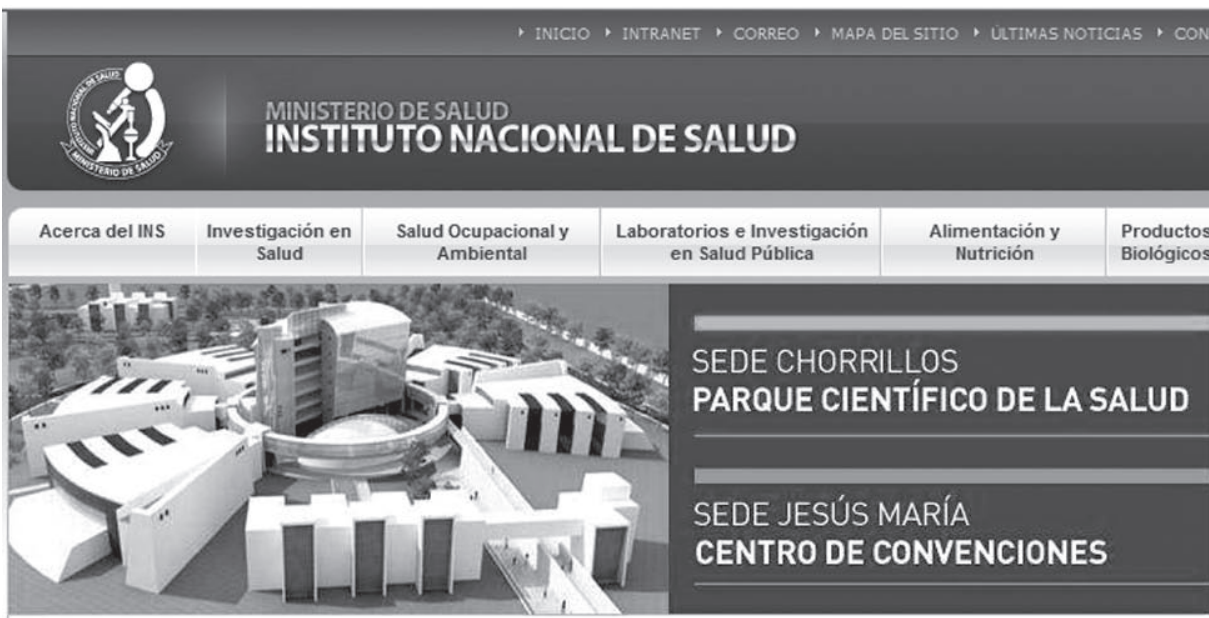

ÚLTIMAS INVESTIGACIONES Y EVIDENCIAS CIENTíFICAS

Distribución de serotipos del virus dengue Perú 2011

Serotipos de dengue identificados por RT-PCR-TR en el Perú 2011

Ver detalle

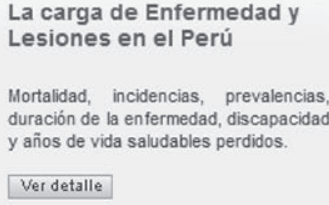

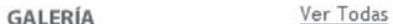

Imagen del dia 2011

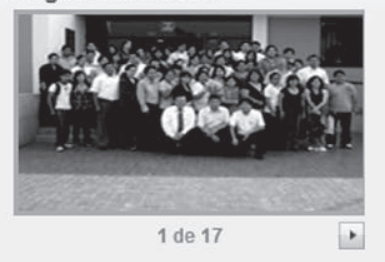

Salud Control de Calidad Intercultural de Medicamentos

Q Portal del Transparencia

- Gestión de calidad

5 Directorio Institucional

12. 30 marzo del 2011

A Agregar a favoritos

- Enviar a un amigo

Publicaciones

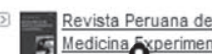
- Medicina Gorimental s Ver más

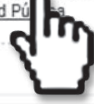

Comités del INS

(2) Comité Especial del Sistema de Control Interno 